\title{
Design and Simulation Analysis of Detection Sensor of Localized Faults for Wire Rope
}

\author{
Bo $\mathrm{Wu}^{\text {a }}$, Guoping $\mathrm{Li}^{\mathrm{b}}$ \\ School of Mechanical Engineering of Jinan University, Jinan 250022, China \\ awb3318866@163.com, b727515509@qq.com
}

Keywords: Wire rope, hall element, localized faults detection, magnetic flux leakage.

\begin{abstract}
The wire rope is widely used in engineering as a flexure component. It is necessary that the wire rope defect must be detected for certain damage would be inevitable occurred in the using process. In this article, the brief introduction about the state of the wire rope defect in the domestic is given. The hall element differential arrangement form has been selected to design the detection sensor of localized faults based on the principle of magnetic flux leakage detection. The wire ropes with different diameters have corresponding excitation structures. Using PDE tool box of MATLAB to simulate the magnetic flux leakage generated from different conditions of broken wires, from which curves of broken wire length and depth influence on the magnetic flux leakage could be received. The corresponding relationships between the magnetic flux leakage and the broken wires have been obtained by analyzing the curves.
\end{abstract}

\section{Introduction}

The wire rope is widely used as a key component in the promotion, transportation and load bearing equipments. It is very important for the use of security. As well, it is extremely essential to detect the damage condition of the wire rope on account of that certain damage would be inevitable occurred in the using process.

Lots of experts and scholars have thoroughly researched the damage detection of the wire rope and received efficient achievements. Including that the MTC-94 and GDJY wire rope detectors were designed by Huazhong University of Science and Technology, which could quantitative identify the broken wires ${ }^{[1]}$. The TGS wire rope detector was concurrently developed by Harbin Institute of Technology and Fushun coal science researching college, which could simultaneously detect LMA and LF damages ${ }^{[2]}$. Qingdao Technological University developed the hall detector could inhibit common-mode interference effectively and the coil detector was suitable for LMA damage ${ }^{[3]}$, etc. At present, quantificational detecting the LF damage is still a difficulty, therefore, it will be of great importance in simulative analyze the LF damage of wire rope from the view of detection principle.

\section{The Detection Principle of the LF Damage for Wire Rope}

The LF damage of wire rope refers to damage produced on the local of wire rope, including broken wires inside and outside, corrosion, and local abnormal shape, etc, which mainly composed by broken wires.

When the wire rope was magnetized to saturation by excitation system, part of magnetic field would be leaked due to reduction of local permeability of the wire rope once the broken wires or other damages were appeared on the surface or inside of wire rope. This leakage magnetic field could be detected by detecting element and then the damage of wire rope would be found ${ }^{[4]}$.

\section{Design of Detection Sensor of LF Damage for Wire Rope}

Hall element has the characteristics of high sensitivity, wide frequency response, huge dynamic range, simple structure, little volume, etc. Therefore, it can be used to detect the LF damage of wire 
rope. Choose hall elements circumferential arrangement because of broken wires or other damages could appear on any circumferential positions.

Many factors have effect on signal output of hall element, such as magnetic fluctuation caused by strand wave of wire rope, magnetic variation caused by lateral oscillation in the process of wire rope moving and random changing electromagnetic radiation at testing ground. Thus, differential arrangement of hall elements with double rows has been chose. Figure 1 is the schematic diagram of hall elements with double rows. The number of hall elements is related to diameter of wire rope.

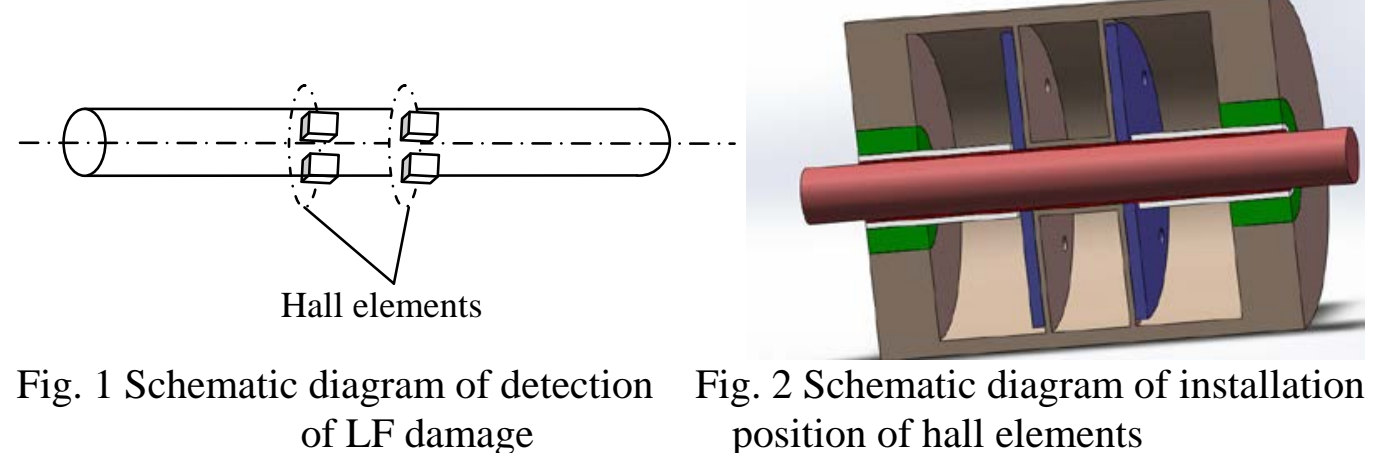

Hall element need to be zero adjusted for its static output characteristic. To make the detection circuit inside of hall sensor to symmetrical semicircle circuit board, then install them to inside of the excitation structure, as shown in figure 2.

\section{Simulation Analysis of Magnetic of LF Damage for Wire Rope}

MATLAB provides PDE tool-box as a graphical user interface. Using this tool box can achieve a variety of functions, such as interactively establishing mathematical model of partial differential equation, setting boundary conditions, generating and refining the triangle mesh, setting the type of partial differential equations, presetting parameters, solving equations and show graphic results ${ }^{[5]}$. Modeling and simulation of leakage magnetic field of LF damage for wire rope can be realized using PDE tool box.

\subsection{Excitation Structure Parameters of Different Diameter of Wire Rope.}

The excitation structure would be increased with the diameter of wire rope increasing. The size of excitation structure must be the minimum size to make wire rope to the saturation magnetization. Size of each part of excitation structures with different diameter of wire rope is shown in table 1.

Table 1 The parameters of excitation structure

\begin{tabular}{ccccccc}
\hline \multicolumn{7}{c}{ The parameters of excitation structure $(\mathrm{mm})$} \\
\hline $\mathrm{D}$ & $\mathrm{Ls}$ & $\mathrm{Tx}$ & $\mathrm{S}$ & $\mathrm{Lm}$ & Tm & $\delta$ \\
5 & 45 & 3 & 30 & 10 & 20 & 2 \\
10 & 55 & 4 & 30 & 10 & 20 & 2 \\
20 & 65 & 4 & 30 & 10 & 20 & 2 \\
30 & 80 & 4 & 40 & 10 & 30 & 2
\end{tabular}

D- diameter of wire rope; $\mathrm{L}_{\mathrm{s}}$ - inside distance of armature iron; $\mathrm{T}_{\mathrm{x}}$ - radial thickness of armature iron; S- distance from nside distance of armature iron to the surface of wire rope; $\mathrm{L}_{\mathrm{m}}$ - axial length of permanent magnet; $\mathrm{T}_{\mathrm{m}}$ - radial length of permanent magnet; $\delta$ - air-gap.

\subsection{Magnetization of the Wire Rope and Simulation Analysis of Leakage Magnetic Field.}

The wire rope is a strong magnetic conductor. Its permeability is 100 times more than the air permeability. The basic principle of magnetic flux leakage testing is based on this.

The air relative permeability is 1.0 , the permanent magnet relative permeability is 1.0 , and the armature iron (mild steel) relative permeability is 1000 .

The typical magnetization curve and permeability curve are shown in figure 3 . In the system of wire rope detection,

$$
\mu=B / H \text {. }
$$


$\mathrm{H}$ - magnetic intensity; B- magnetic induction; ${ }^{\mu}$ - the wire rope permeability.

Because of B-H curve has the characteristics of nonlinearity and $\mu$ is changeable. As shown in figure 3 , when the wire rope is magnetized saturation, $B>=1 T$, this moment the wire rope relative permeability is 100 .

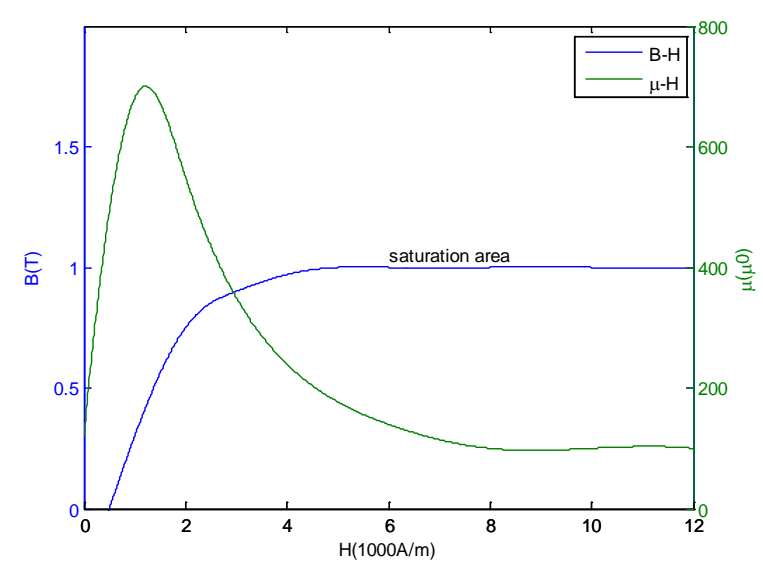

Fig. 3 The typical magnetization curve of wire rope

The excitation structure is composed of wire rope, permanent magnet, armature iron and air-gap. Use different rectangle with different parameter expresses the components above. Make the wire rope with $5 \mathrm{~mm}$ diameter for example. The simulation steps as shown in figure 4.

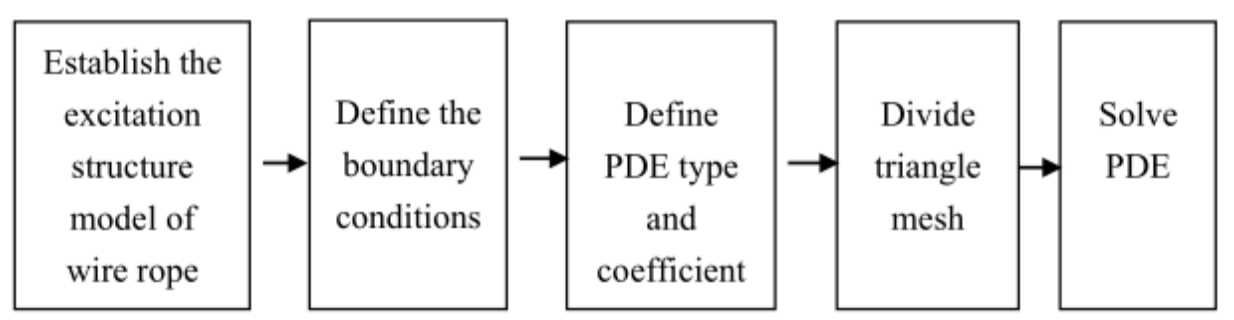

Fig. 4 Steps of using MATLAB to analyze excitation magnetic field of wire rope Simulation result can be obtained from steps above, as shown in figure 5.

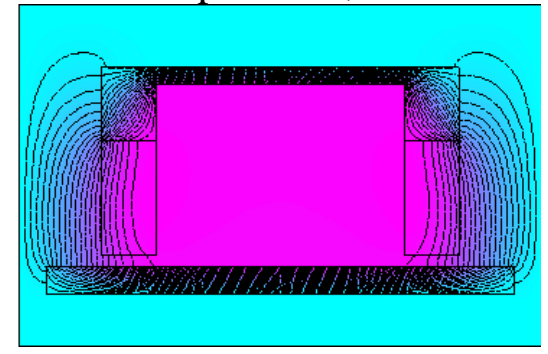

Fig. 5 Simulation result

\subsection{Simulation Analysis of Leakage Magnetic Field of Broken Wires.}

Set value range of abscissa and ordinate as [-50 50] (mm), place different rectangles to express different level of broken wires and simulate broken wires of wire rope on different conditions, as shown in figure 6.

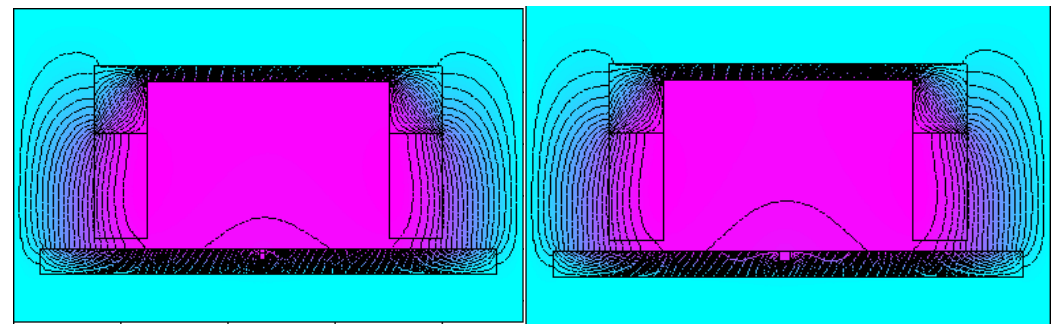

(a) $1 \mathrm{~mm}$ length, $2 \mathrm{~mm}$ depth $\quad$ (b) $2 \mathrm{~mm}$ length, $2 \mathrm{~mm}$ depth 


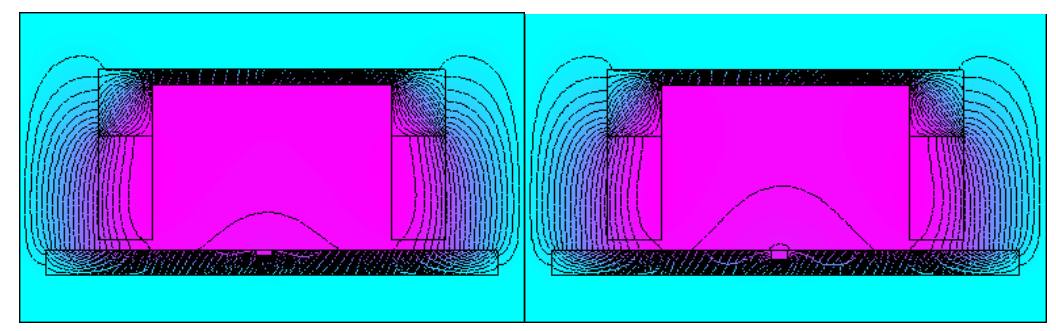

(c) 3mm length, $1 \mathrm{~mm}$ depth (d) $3 \mathrm{~mm}$ length, $2 \mathrm{~mm}$ depth

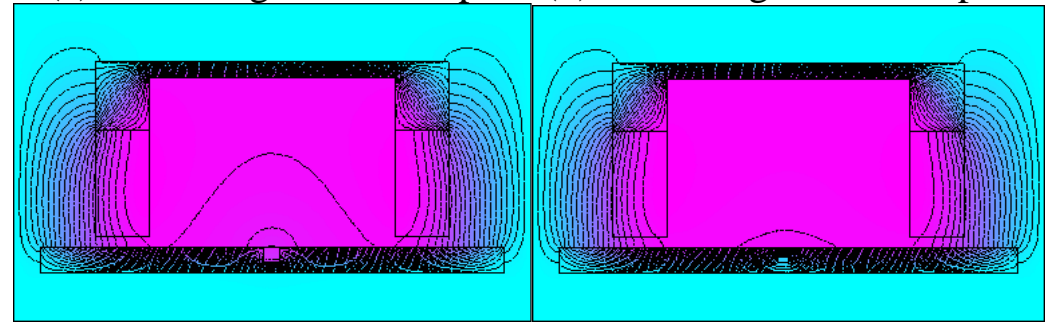

(e) 3mm length, 3mm $\quad$ (f) internal broken wires

Fig. 6 Simulation results of broken wires

(1) Influence of broken wire length: select broken wires depth as $2 \mathrm{~mm}$, length as $1-7 \mathrm{~mm}$, read the corresponding outermost numerical value of the peak point of leakage flux waveform, as shown in table 2. Draw the curve of the peak point of leakage flux waveform changing with broken wires length, as shown in figure 7 . Simulate the changeable peak of leakage magnetic field on different conditions of broken wires length.

Table 2 Value point with different broken wires length

The broken wires length/mm

$\begin{array}{lllllll}1 & 2 & 3 & 4 & 5 & 6 & 7\end{array}$

The peak point of leakage flux waveform

$\begin{array}{lllllll}7 & 9.5 & 12 & 15 & 19 & 24 & 32\end{array}$

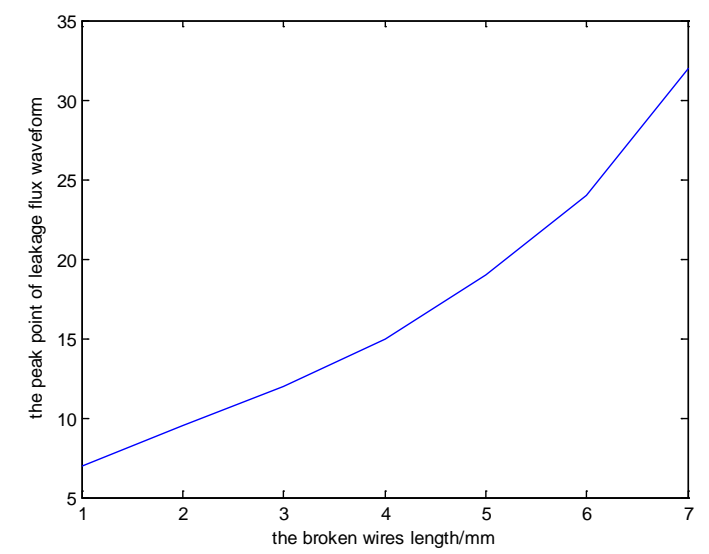

Fig. 7 The curve of broken wires length influence on leakage magnetic flux

(2) Influence of broken wire depth: select broken wires length as 3mm, depth as $0.5-3.5 \mathrm{~mm}$, read the corresponding outermost numerical value of the peak point of leakage flux waveform, as shown in table 3. Draw the curve of the peak point of leakage flux waveform changing with broken wires depth, as shown in figure 8 . Simulate the changeable peak of leakage magnetic field on different conditions of broken wires depth.

Table 3 Value point with different broken wires depth

The broken wires depth/mm

$\begin{array}{lllllll}0.5 & 1 & 1.5 & 2 & 2.5 & 3 & 3.5\end{array}$

The peak point of leakage flux waveform

$\begin{array}{lllllll}4.5 & 8 & 10 & 12 & 15 & 18 & 21\end{array}$




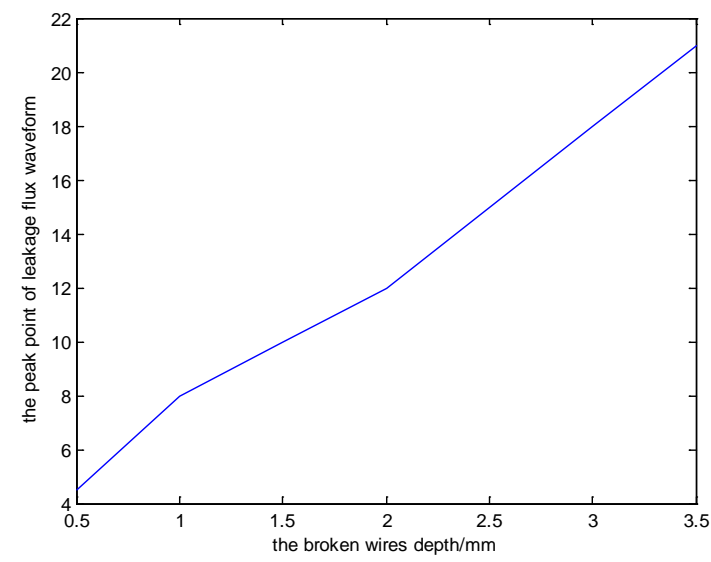

Fig. 8 The curve of broken wires depth influence on leakage magnetic flux

As can be seen from figure 6(a)-(e), the leakage magnetic field generated from external broken wires has relationship with the length and depth of broken wires. The leakage flux density and leakage magnetic field would be bigger along with the length and depth of broken wires become bigger. From figure 6(f), it can be seen that internal broken wires can also cause magnetic field to give away. Therefore, using sensor can detect the leakage magnetic field generated from internal and external broken wires. Through detecting the size of signal can judge the quantity of broken wires.

\section{Summary}

This paper introduced design of detection sensor of localized faults for wire rope from aspects that the principle of magnetic flux leakage detection and simulation analysis of magnetic flux leakage. Using PDE tool-box of MATLAB to analyze the leakage magnetic field of wire rope damages gets the following conclusions: according to the changeable leakage flux signals to analyze the conditions of broken wires, based on the LF damage detection of wire rope leakage magnetic field The characteristic of the axial and radial leakage magnetic flux is different, from arranging direction of hall elements to separated detect.

\section{References}

[1]. Jianhua Jin, Yihua Kang. Method of decision fusion of multi-sensor information and the applications in the electromagnetic testing. Nondestructive Testing. Vol. 25 (2003) No. 11, p. 585-588.

[2]. Chong Chen, Jinqi Liu, Dianguo Xu. Intelligent steel wire rope flaw detector. Nondestructive Detection. (1994) No.6, p. 33-36.

[3]. Jiwen Tan, et al. The principle and technology of wire rope safety inspection. Publishing House of Science, 2009.

[4]. Hua Liang, Ruyu Hong, Guangxiang Yang. Research of wire rope inspection technology based on leakage flux signals. Piezoelectrics \& Acousto-optics. Vol. 30 (2008) No.4, p. 508-510.

[5]. Jinming Su, Lianhua Zhang, Bo Liu, et al. Application of MATLAB tool box. Publishing House of Electronics Industry, 2004. 